\title{
HER2 screening data from ToGA: targeting HER2 in gastric and gastroesophageal junction cancer
}

\author{
Eric Van Cutsem • Yung-Jue Bang $\cdot$ Feng Feng-yi $\cdot$ Jian M. Xu $\cdot$ Keun-Wook Lee $\cdot$ Shun-Chang Jiao \\ Jorge León Chong $\cdot$ Roberto I. López-Sanchez $\cdot$ Timothy Price $\cdot$ Oleg Gladkov $\cdot$ Oliver Stoss $•$ \\ Julie Hill $\cdot$ Vivian Ng $\cdot$ Michaela Lehle $\cdot$ Marlene Thomas $\cdot$ Astrid Kiermaier $\cdot$ Josef Rüschoff
}

Received: 26 March 2014/ Accepted: 11 June 2014/Published online: 20 July 2014

(C) The Author(s) 2014. This article is published with open access at Springerlink.com

\begin{abstract}
Background In the Trastuzumab for GAstric cancer (ToGA) study, trastuzumab plus chemotherapy improved median overall survival by 2.7 months in patients with human epidermal growth factor receptor 2 (HER2)-positive [immunohistochemistry (IHC) 3+/fluorescence in situ hybridization-positive] gastric/gastroesophageal junction cancer compared with chemotherapy alone (hazard ratio 0.74). Post hoc exploratory analyses in patients expressing higher HER2 levels (IHC 2+/fluorescence in situ hybridization-positive or IHC $3+$ ) demonstrated a 4.2-month
\end{abstract}

E. Van Cutsem and Y.-J. Bang contributed equally.

Julie Hill: Work undertaken at Roche Products Pty Ltd., Dee Why, NSW, Australia.

Electronic supplementary material The online version of this article (doi:10.1007/s10120-014-0402-y) contains supplementary material, which is available to authorized users.

E. Van Cutsem $(\square)$

University Hospitals Leuven and KU Leuven, Leuven, Belgium

e-mail: eric.vancutsem@uzleuven.be

Y.-J. Bang

Seoul National University College of Medicine, Seoul, South

Korea

F. Feng-yi

Cancer Institute and Hospital, Beijing, China

J. M. Xu

Affiliated Hospital (307 Hospital) Cancer Centre,

Beijing, China

K.-W. Lee

Seoul National University Bundang Hospital, Seoul National

University College of Medicine, Seongnam, South Korea improvement in median overall survival with trastuzumab (hazard ratio 0.65). The ToGA study provides the largest screening dataset available on HER2 overexpression/ amplification in this indication. We further analyzed correlation(s) of HER2 overexpression/amplification with clinical and epidemiological factors.

Methods HER2-positivity was analyzed by histological subtype, tumor location, geographic region, and specimen type. Exploratory efficacy analyses were performed.

Results The HER2-positivity rate was $22.1 \%$ across analyzed tumor samples. Rates were similar between European and Asian patients $(23.6 \%$ vs. $23.9 \%)$, but higher in intestinal- vs. diffuse-type ( $31.8 \%$ vs. $6.1 \%$ ), and gastroesophageal junction cancer versus gastric tumors (32.2\% vs. $21.4 \%)$. Across all IHC scores, variability in HER2 staining ( $\leq 30 \%$ stained cells) was observed in almost $50 \%$ of cases, with increasing rates in lower IHC categories, and did not affect treatment outcome. The polysomy rate was $4 \%$.

S.-C. Jiao

General Hospital of P.L.A., Beijing, China

J. L. Chong

Instituto Nacional de Enfermedades Neoplásicas, Lima, Peru

R. I. López-Sanchez

Centro Oncológico Punta Pacífica, Panama City, Panama

T. Price

The Queen Elizabeth Hospital, Woodville, SA, Australia

O. Gladkov

Regional Oncology Dispensary, Chelyabinsk, Russian

Federation

O. Stoss $\cdot$ J. Rüschoff

Targos Molecular Pathology GmbH, Kassel, Germany 
Conclusions HER2 expression varies by tumor location and type. All patients with advanced gastric or gastroesophageal junction cancer should be tested for HER2 status, preferably using IHC initially. Due to the unique characteristics of gastric cancer, specific testing/scoring guidelines should be adhered to.

Keywords Gastric cancer - HER2 testing · Immunohistochemistry · In situ hybridization . Trastuzumab

\section{Introduction}

Human epidermal growth factor receptor 2 (HER2) is a key driver of tumorigenesis [1], where it is implicated in tumor cell proliferation, apoptosis, adhesion, migration, and differentiation [2]. HER2 has been validated as a prognostic and predictive factor in breast cancer [1,3], and evidence is growing that HER2 is also a driver of tumorigenesis in gastric and gastroesophageal junction cancer, with studies suggesting that HER2 amplification or overexpression is relatively frequent in this tumor type [2, 4-6]. Studies of HER2-positivity rates in gastric cancer using immunohistochemistry (IHC) and fluorescence or chromogenic in situ hybridization (FISH/CISH) have shown broad variations, ranging from $6.8 \%$ to $34.0 \%$ for IHC [7, 8], $7.1 \%$ to $42.6 \%$ for FISH [7, 9], and $12.2 \%$ to $24.0 \%$ for $\mathrm{CISH}$ [6]. Such high variability in HER2-positivity rates can partly be explained by the fact that early reported HER2 data were generated using the breast cancer HER2 testing and/or scoring principles, or were performed with nonvalidated tests. Differences in tumor histology and tumor locations between different cohorts studied may also contribute to the observed high variability of HER2-positivity reported in the literature. Although it remains to be fully established whether HER2 is a valid prognostic factor in

\section{J. Hill}

Roche Products Pty Ltd., Dee Why, NSW, Australia

Present Address:

J. Hill

McCloud Consulting Group, Gordon, NSW, Australia

V. Ng

Genentech Inc., South San Francisco, CA, USA

M. Lehle

F. Hoffmann-La Roche Ltd., Basel, Switzerland

M. Thomas

Roche Diagnostics GmbH, Penzberg, Germany

A. Kiermaier

Genentech Inc., Basel, Switzerland gastric cancer, some studies have suggested that HER2positivity is associated with poor outcomes and more aggressive disease $[2,5,6,10]$, whereas others have shown HER2 to be a favorable prognostic factor [11].

All patients assessed for enrollment into the international, randomized, phase III Trastuzumab for GAstric cancer (ToGA) study were tested for HER2 overexpression and amplification by IHC and FISH, respectively [12]. Patients with advanced gastric or gastroesophageal junction tumors that had HER2 overexpression (IHC 3+) and/ or gene amplification (FISH-positive) were eligible for enrollment into the study, if all other inclusion criteria were met, and randomized to trastuzumab (Herceptin ${ }^{\circledR}, \mathrm{F}$. Hoffmann-La Roche Ltd., Basel, Switzerland) plus chemotherapy or to chemotherapy alone [12]. Bang and Van Cutsem et al. described the results of this phase III study, showing that trastuzumab in combination with chemotherapy significantly prolonged survival compared with chemotherapy alone in patients with advanced gastric or gastroesophageal junction tumors that showed HER2 overexpression or gene amplification [median overall survival: 13.8 months vs. 11.1 months, respectively; hazard ratio (HR) 0.74 ; $95 \%$ confidence interval (CI), 0.60-0.91; $P=0.0046]$ [12]. A post hoc exploratory analysis showed a median overall survival of 16.0 months in a subset of patients receiving trastuzumab plus chemotherapy (vs. 11.8 months for patients receiving chemotherapy alone) whose tumors showed higher HER2 protein overexpression (IHC $2+/$ FISH-positive or IHC $3+$ ), with evidence of a significant interaction test between treatment and the two subgroups of high vs. low HER2 expression $(P=0.036$ using the log-likelihood ratio test) [12]. These data emphasize the need for high-quality, accurate, and standardized HER2 testing to ensure identification of the patient population that will benefit from trastuzumab.

Here, we present key HER2 screening results from the ToGA study, including data on IHC and FISH testing from the largest population of patients with gastric or gastroesophageal junction tumors to date, and HER2 testing data according to different patient and tumor characteristics. Efficacy data according to HER2 test results are also reported.

\section{Materials and methods}

Study design and HER2 screening

The study design for ToGA (Clinical trials.gov ID\#: NCT01041404: http://clinicaltrials.gov/ct2/show/NCT0104 1404) has been described previously [12]. Briefly, patients with advanced gastric or gastroesophageal junction cancer displaying HER2 overexpression or amplification were randomized to trastuzumab plus chemotherapy 
Table 1 HER2 testing results from the screening phase of the study, according to immunohistochemistry and fluorescence in situ hybridization

\begin{tabular}{llllll}
\hline Total screening population $^{\mathrm{a}}(N=3,280)$ & IHC 0 & IHC 1+ & IHC 2+ & IHC 3+ & Total \\
\hline FISH-positive & $94^{\mathrm{b}}(4.9)$ & $96(15.7)$ & $212(54.6)$ & $354(94.9)$ & $756(23.0)$ \\
FISH-negative & $1,815^{\mathrm{b}}(95.1)$ & $514(84.3)$ & $176(45.4)$ & $19(5.1)$ & $2,524(77.0)$ \\
Total & $1,909(100)$ & $610(100)$ & $388(100)$ & $373(100)$ & $3,280(100)$ \\
\hline
\end{tabular}

Values are expressed as $n(\%)$

FISH, fluorescence in situ hybridization; HER2, human epidermal growth factor receptor 2; IHC, immunohistochemistry

a With both IHC and FISH results

${ }^{\mathrm{b}}$ One patient originally classified as FISH-positive/IHC 0 was reclassified as FISH-negative/IHC 0 after 1 year's follow-up but was included in the intention-to-treat analyses (HER2-positive)

Table 2 HER2 testing results in patients potentially eligible for enrollment into ToGA, according to immunohistochemistry and fluorescence in situ hybridization

\begin{tabular}{llllll}
\hline $\begin{array}{l}\text { Patients eligible for ToGA based } \\
\text { on HER2 status }(N=810)\end{array}$ & IHC 0 & IHC & IHC & IHC \\
1+ & & $94^{\mathrm{a}}(11.6)$ & $96(11.9)$ & $212(26.2)$ & $\begin{array}{l}\text { No } \\
\text { IHC }\end{array}$ \\
\hline FISH-positive & - & - & - & $154(43.7)$ & $19(2.3)$ \\
FISH-negative & - & - & - & $25(3.1)$ & - \\
No FISH result & & - & - \\
\hline
\end{tabular}

Values are expressed as $n(\%)$

FISH, fluorescence in situ hybridization; HER2, human epidermal growth factor receptor 2; IHC, immunohistochemistry; ToGA, Trastuzumab for GAstric cancer

a One patient originally classified as FISH-positive/IHC 0 was reclassified as FISH-negative/IHC 0 after 1 year's follow-up but was included in the intention-to-treat analyses (HER2-positive)

[capecitabine (Xeloda ${ }^{\circledR}$, F. Hoffmann-La Roche Ltd.) or 5-fluorouracil plus cisplatin (XP/FP)] or to chemotherapy alone. All patients provided written informed consent. Investigators performed the human investigations after approval by a Local Human Investigations Committee. HER2 testing was performed in a central laboratory (Targos Molecular Pathology GmbH, Kassel, Germany) by IHC [HercepTest $^{\mathrm{TM}}$ (Dako, Glostrup, Denmark)] and FISH [HER2 FISH pharmDx ${ }^{\mathrm{TM}}$ (Dako)] in parallel, and patients whose tumors scored IHC $3+$ or had a HER2:CEP17 ratio of $\geq 2.0$ by FISH were considered eligible for the study. IHC samples were scored according to the criteria specifically developed for gastric cancer [4], which were applied to the ToGA trial as described by Bang and Van Cutsem et al. [12].

At the screening stage, samples were also classified according to clinical parameters including histological subtype according to the Lauren classification [13] (intestinal, diffuse, or mixed), location of the tumor specimen received (stomach or gastroesophageal junction) and specimen type (biopsy or surgical). Post hoc subgroup analyses for efficacy parameters were performed in two subgroups: patients whose tumors showed lower levels of HER2 protein expression (IHC 0/FISH-positive or IHC 1+/FISH-positive) and those with higher levels of HER2 protein expression [IHC $3+$ (including IHC $3+/$ FISHnegative, IHC $3+/$ FISH no result, and IHC $3+/$ FISHpositive) or IHC 2+/FISH-positive].
Efficacy was also assessed in terms of tumor heterogeneity, which was defined as variability in IHC staining in our analysis. We describe two subgroups based on a cutoff of $\leq 30 \%$ of tumor cells stained vs. $>30 \%$ of tumor cells stained [14].

\section{Statistical analyses}

Post hoc exploratory analyses were performed using the statistical program R, Version 1.9.1, and Microsoft Excel. Time-to-event endpoints were analyzed using a Cox regression model. Subgroup analyses were summarized by median values, HRs, and corresponding $95 \%$ CIs. The interaction between treatment and subgroups was assessed using the log-likelihood ratio test. For the analysis of overall tumor response, the proportion of responders in each treatment group (and the two-sided $95 \%$ PearsonClopper CIs), together with the odds ratio, is presented.

\section{Results}

HER2 in advanced gastric and gastroesophageal junction cancer

A total of 3,803 patients were screened for HER2 status; either IHC or FISH testing was successful in 3,665 patients 
Table 3 Proportion of tumors that tested positive for HER2 based on data from all patients screened and from patients successfully screened by immunohistochemistry or fluorescence in situ hybridization, according to patient demographics and tumor characteristics

\begin{tabular}{|c|c|c|c|}
\hline & $\begin{array}{l}\text { All } \\
\text { patients }^{\mathrm{a}}\end{array}$ & $\begin{array}{l}\text { IHC 3+ } \\
\text { or FISH- } \\
\text { positive }\end{array}$ & $\begin{array}{l}\text { HER2- } \\
\text { positivity } \\
\text { rate } n / N^{\mathrm{b}}(\%)\end{array}$ \\
\hline All patients screened & 3,803 & 810 & $\mathrm{n} / \mathrm{a}$ \\
\hline $\begin{array}{l}\text { Successfully screened } \\
\text { by IHC or FISH }\end{array}$ & 3,665 & 810 & 22.1 \\
\hline \multicolumn{4}{|l|}{ Specimen location } \\
\hline Stomach & $2,195(58)$ & $451(56)$ & $451 / 2,112(21.4)$ \\
\hline $\begin{array}{l}\text { Gastroesophageal } \\
\text { junction }\end{array}$ & $208(5)$ & $65(8)$ & $65 / 202(32.2)$ \\
\hline Metastatic sites & $322(8)$ & $51(6)$ & $51 / 305(16.7)$ \\
\hline $\begin{array}{l}\text { Primary tumor (exact } \\
\text { location of } \\
\text { specimen not } \\
\text { recorded) }\end{array}$ & $1,046(28)$ & $232(29)$ & $232 / 1,017(22.8)$ \\
\hline Not assessed & $32(<1)$ & $9(1)$ & $9 / 29(31.0)$ \\
\hline \multicolumn{4}{|l|}{ Tumor subtype } \\
\hline Diffuse & $1,117(29)$ & $68(8)$ & $68 / 1,108(6.1)$ \\
\hline Intestinal & $1,916(50)$ & $606(75)$ & $606 / 1,904(31.8)$ \\
\hline Mixed & $652(17)$ & $131(16)$ & $131 / 650(20.0)$ \\
\hline Not assessed & $118(3)$ & $5(<1)$ & $5 / 118(4.2)$ \\
\hline \multicolumn{4}{|l|}{ Specimen type } \\
\hline Biopsy & $2,596(68)$ & $579(71)$ & $579 / 2,492(23.2)$ \\
\hline Surgical & $1,199(32)$ & $231(29)$ & $231 / 1,173(19.7)$ \\
\hline Not assessable & $8(<1)$ & $0(0)$ & 0/0 (0.0) \\
\hline \multicolumn{4}{|l|}{ Region of origin $^{c}$} \\
\hline Asia-Pacific & $1,900(52)$ & $454(56)$ & $454 / 1,900(23.9)$ \\
\hline Europe & $795(22)$ & $188(23)$ & 188/795 (23.6) \\
\hline $\begin{array}{l}\text { Central/South } \\
\text { America }\end{array}$ & $484(13)$ & $78(10)$ & 78/484 (16.1) \\
\hline Other $^{\mathrm{d}}$ & $486(13)$ & $89(11)$ & $89 / 486(18.3)$ \\
\hline
\end{tabular}

Values are expressed as $n(\%)$

FISH, fluorescence in situ hybridization; HER2, human epidermal growth factor receptor 2; IHC, immunohistochemistry

${ }^{a}$ As a proportion of the 3,803 patients screened

b As a proportion of the 3,665 patients successfully screened according to study protocol, where HER2-positivity was defined as IHC 3+ or FISHpositive [for region of origin, HER2-positivity is calculated based on all patients screened $(N=3,803)$ ]

${ }^{c}$ Region-of-origin analysis relates to 809 patients who were IHC $3+$ or FISH-positive; one patient was reclassified as FISH-negative/IHC 0 after 1 year's follow-up but was included in the intention-to-treat analyses (HER2-positive)

${ }^{\mathrm{d}}$ Russia and South Africa

[12]. Results for both IHC and FISH were available from 3,280 patients, and concordance between the two methodologies was calculated with defined categories of IHCpositive (IHC 3+), IHC-negative (IHC 0 , IHC 1+, and IHC 2+), FISH-positive, and FISH-negative. Concordance
Table 4 Proportion of patients with tumor HER2 staining variability ( $\leq 30 \%$ stained cells) by IHC category

\begin{tabular}{llll}
\hline $\begin{array}{l}\text { Patients with } \leq 30 \% \\
\text { stained cells, \% }\end{array}$ & $\begin{array}{l}\text { Screening } \\
\text { population }\end{array}$ & $\begin{array}{l}\text { Randomized and } \\
\text { treated population }\end{array}$ \\
\hline IHC 1+ & 88.5 & 85.7 & \\
IHC 2+ & 68.5 & 54.7 & \\
IHC 3+ & 30.5 & 29.9 & \\
\hline $\begin{array}{l}\text { HER2, human epidermal } \\
\text { immunohistochemistry }\end{array}$ & growth factor & receptor 2; IHC,
\end{tabular}

between IHC and FISH with the categories defined above was $87.2 \%(2,859 / 3,280$ cases $)$; a total of $354 / 3,280$ samples $(10.8 \%)$ were IHC-positive and FISH-positive, and 2,505/3,280 (76.4\%) samples were scored as IHCnegative and FISH-negative (Table 1). A total of $7.5 \%$ $(190 / 2,519)$ of IHC $0 / 1+$ cases and $74.4 \%(566 / 761)$ of IHC $2+/ 3+$ cases scored FISH-positive (Table 1). Notably, among patients whose tumors scored IHC 3+, $94.9 \%$ (354/373) were FISH-positive, while $54.6 \%(212 / 388)$ were FISH-positive in the IHC $2+$ group (Table 1). As the IHC $2+$ category represents a subgroup of patients with an equivocal result, this subgroup was excluded from further exploratory analyses of concordance. By excluding IHC $2+$ samples, concordance between IHC and FISH was $92.8 \%(2,683 / 2,892)$ (Table 1).

In total, 810/3,665 (22.1\%) successfully tested patients had IHC $3+$ or FISH-positive tumors and were potentially eligible for enrollment (Table 2). Among these 810 patients, $3.1 \%$ (25 patients) had no FISH result available; $1.2 \%$ (10 patients) had no IHC result available; approximately half (398 patients; $49.1 \%$ ) had tumors that were IHC $3+$, and the remainder were equally distributed between IHC 0/1+ [190 patients; IHC 0 (94 patients) and IHC $1+$ (96 patients); $23.5 \%$ ] and IHC $2+$ (212 patients; $26.2 \%$; Table 2).

HER2 overexpression/gene amplification rates were similar across patients from Europe and Asia (23.6 \% vs. $23.9 \%$; Table 3), but slightly lower in those from Central/ South America (16.1\%).

Overexpression or amplification of HER2 was more common in patients with intestinal histology compared with those with diffuse histology $(31.8 \%$ vs. $6.1 \%$, respectively; Table 3) and in specimens from the gastroesophageal junction compared with specimens from the stomach (32.2 \% vs. $21.4 \%$, respectively; Table 3$)$. There was also a slightly higher rate of HER2 overexpression/ amplification in biopsy specimens compared with surgical specimens (23.2 \% vs. $19.7 \%$, respectively; Table 3 ).

Variability in HER2 staining intensity $(\leq 30 \%$ positively stained cells) occurred in 294/584 (50.3\%) patients across all IHC categories in all randomized and treated patients. For IHC $3+$ samples, $3.1 \%$ (biopsies only), $26.8 \%, 31.0 \%$, and $39.0 \%$ of cases showed HER2 
Table 5 Efficacy of trastuzumab plus chemotherapy vs. chemotherapy alone according to HER2 expression status (screened by immunohistochemistry or fluorescence in situ hybridization)

Seven patients did not have an IHC score and were therefore excluded

CI, confidence interval; FISH, fluorescence in situ hybridization; HER2, human epidermal growth factor receptor 2; IHC, immunohistochemistry; ToGA, Trastuzumab for GAstric cancer; XP/FP, capecitabine plus cisplatin or 5 -fluorouracil plus cisplatin

${ }^{\text {a }}$ Overall survival in the ToGA population was 11.1 months for chemotherapy alone and 13.8 months for the trastuzumab plus chemotherapy arm (HR 0.74; $95 \%$ CI, 0.60-0.91) [12]

\begin{tabular}{|c|c|c|c|c|}
\hline & \multicolumn{2}{|c|}{$\begin{array}{l}\text { Lower HER } 2 \text { expression (IHC 0/FISH- } \\
\text { positive or IHC } 1+/ \text { FISH-positive) }\end{array}$} & \multicolumn{2}{|c|}{$\begin{array}{l}\text { Higher HER } 2 \text { expression (IHC } 2+\text { l } \\
\text { FISH-positive or IHC } 3+\text { ) }\end{array}$} \\
\hline & $\begin{array}{l}\mathrm{XP} / \mathrm{FP} \\
(n=70)\end{array}$ & $\begin{array}{l}\text { Trastuzumab }+\mathrm{XP} / \mathrm{FP} \\
(n=61)\end{array}$ & $\begin{array}{l}\mathrm{XP} / \mathrm{FP} \\
(n=218)\end{array}$ & $\begin{array}{l}\text { Trastuzumab }+\mathrm{XP} / \mathrm{FP} \\
(n=228)\end{array}$ \\
\hline \multicolumn{5}{|l|}{ Overall survival $^{\mathrm{a}}[12]$} \\
\hline Median (months) & 8.7 & 10.0 & 11.8 & 16.0 \\
\hline Hazard ratio & 1.07 & & 0.65 & \\
\hline $95 \% \mathrm{CI}$ & $0.70-1.62$ & & $0.51-0.83$ & \\
\hline \multicolumn{5}{|c|}{ Progression-free survival } \\
\hline Median (months) & 4.8 & 5.3 & 5.5 & 7.6 \\
\hline Hazard ratio & 1.00 & & 0.64 & \\
\hline $95 \% \mathrm{CI}$ & $0.69-1.45$ & & $0.51-0.79$ & \\
\hline \multicolumn{5}{|l|}{ Time to progression } \\
\hline Median (months) & 5.1 & 5.5 & 5.7 & 7.9 \\
\hline Hazard ratio & 1.00 & & 0.64 & \\
\hline $95 \% \mathrm{CI}$ & $0.68-1.47$ & & $0.51-0.80$ & \\
\hline \multicolumn{5}{|l|}{ Duration of response } \\
\hline Median (months) & 4.5 & 5.4 & 4.9 & 7.0 \\
\hline Hazard ratio & 0.77 & & 0.5 & \\
\hline $95 \% \mathrm{CI}$ & $0.39-1.52$ & & $0.36-0.71$ & \\
\hline \multicolumn{5}{|l|}{ Tumor response } \\
\hline $\begin{array}{l}\text { Objective response } \\
\text { rate, } n(\%)\end{array}$ & $22(31.4)$ & $21(34.4)$ & $77(35.3)$ & $117(51.3)$ \\
\hline Odds ratio & 1.15 & & 1.93 & \\
\hline $95 \% \mathrm{CI}$ & $0.55-2.38$ & & $1.32-2.82$ & \\
\hline
\end{tabular}

reactivity in $<10 \%, 10$ to $30 \%, 31$ to $79 \%$, and 80 to $100 \%$ of tumor cells, respectively. A comparison of the proportion of samples with $\leq 30 \%$ stained cells per IHC category is shown in Table 4. Variability was greatest in the lower IHC categories. Analysis of the CEP17 signal in tumor samples from evaluable patients showed that polysomy (defined as $\geq 3$ CEP17 signals) occurred in only $23 / 567(4.1 \%)$ of cases in patients randomized and enrolled into ToGA with HER2-overexpressing or gene amplification status and in 133/3,317 (4.0\%) of the entire screening population. Twenty-eight out of the 133 cases showed HER2 gene amplification with a HER2:CEP17 ratio of $\geq 2$, and 11 of these cases also demonstrated an IHC $3+$ score. The remaining 105 polysomic cases had a FISH ratio $<2$; only three of these cases were IHC $3+$. Seven cases had a gene count of $\geq 3$ and FISH ratio of $<2$ : four scoring IHC 0 and one each scoring IHC $1-3+$.

Correlation between HER2 expression level and response to trastuzumab

Previously reported findings showed a greater overall survival benefit of trastuzumab plus chemotherapy in the subgroup of patients with higher HER2 expression levels
[12]. Trastuzumab plus chemotherapy significantly improved all time-to-event endpoints and the objective response rate vs. chemotherapy alone in this analysis (Table 5).

Variability in staining intensity did not adversely affect the overall survival benefit of trastuzumab, although the benefit was numerically lower for patients with tumors exhibiting variable staining, with overlapping CIs (HR $0.84 ; 95 \%$ CI, $0.63-1.11$ vs. HR $0.62 ; 95 \%$ CI, 0.45-0.86) (please see Online resource 1 ). When variability in staining intensity was explored by IHC category, patients with IHC 2+ and IHC 3+ scores showed a treatment benefit regardless of variability, with a numerical trend toward greater benefit in patients with $>30 \%$ cells stained. In the IHC $2+$ category, the HRs were $0.83(95 \%$ CI, 0.50-1.41) in the $\leq 30 \%$ subgroup and $0.66(95 \% \mathrm{CI}$, $0.36-1.18)$ in the $>30 \%$ subgroup. Similarly, in the IHC $3+$ subgroup, HRs were 0.71 (95\% CI, 0.40-1.25) and $0.55(95 \% \mathrm{CI}, 0.37-0.81)$ in the $\leq 30 \%$ and $>30 \%$ subgroups, respectively. Again, CIs were wide and overlapping, and the sizes of subgroups compared within the IHC $3+$ group were imbalanced $(n=86$ and 201). Therefore, firm conclusions could not be drawn. Within the IHC 0 and IHC $1+$ groups, it was not possible to explore 
an impact of tumor heterogeneity on treatment benefit as the subgroup with $>30 \%$ cells stained was small $(n=0$ and 10 , respectively).

Correlation between HER2 amplification level and response to trastuzumab

The HER2 gene copy number $(<6$ or $\geq 6)$ did not affect overall survival in the subgroups of patients with IHC $0 / 1+$ or IHC $2+$ scores, either by stratified or unstratified analyses $(P>0.05$ in all cases).

\section{Discussion}

Advanced gastric cancer is a difficult-to-treat disease for which there is currently no globally accepted standard of care and for which improved treatments are required [15, 16]. HER 2 has been identified as a predictive biomarker in gastric cancer, and efficacy outcomes analyzed in ToGA correlate with the level of HER2 overexpression [12]. Therefore, accurate and standardized HER2 testing is crucial to identify the target population for trastuzumab treatment.

Data from the 3,665 patients who were successfully screened by either IHC or FISH for this study confirm previous reports that HER2 overexpression or amplification is more common in patients with intestinal-type tumors compared with diffuse- or mixed-type tumors [5, 6]. Similarly, our data are consistent with those published elsewhere, suggesting that HER2-positivity rates are higher in specimens from the gastroesophageal junction compared with specimens from the body of the stomach [6, 17]. These differences may be related, as gastroesophageal junction cancers are generally of the intestinal type [18, 19]. These findings suggest that the etiology and pathogenesis of gastroesophageal junction cancer are likely different from those of distal gastric cancer. We also evaluated the relationship between the method of specimen collection (biopsy or surgery) and HER2 overexpression or amplification, and we found that the rates were numerically higher in biopsy samples, although the difference was relatively small (23.2\% vs. $19.7 \%$, respectively) and unlikely to be clinically relevant. One possible explanation for the higher HER2-positivity rate observed in biopsy specimens compared with surgical specimens could be the different cutoff used to define HER2-positivity by IHC (a cluster of five or more tumor cells for biopsies compared with $\geq 10 \%$ of tumor cells for surgical specimens). Another could be that too few biopsy samples were collected from patients, as staining variability was fairly common and may have led to inaccurate results.
The difference in HER2-positivity rates between Europe/Asia and Central/South America may have reflected the substantially lower number of patients screened in Central/South America and does not necessarily reflect a different biology.

Among the total screened population with successful IHC or FISH results, $22.1 \%$ displayed HER2 overexpression or amplification (IHC $3+$ or FISH-positive and therefore eligible for study entry), while $16.6 \%$ were HER2-positive when applying the definition of higher HER2 overexpression (IHC 2+/FISH-positive or IHC 3+). HER2 screening in the ToGA study was performed in a central testing laboratory (Targos Molecular Pathology $\mathrm{GmbH}$ ); it is anticipated that HER2-positivity rates may vary when HER2 testing data are reported from regional centers, further highlighting the need for standardized HER2 testing and scoring and the use of accurate and validated assays [14]. Polysomy seems to play a minor role in gastric cancer; in the ToGA study the observed polysomy rate was very low at around $4 \%$, and very few patients were scored as HER2-negative due to polysomy.

Compared with breast tumors, gastric tumors show higher variability in staining intensities across tissue sections, with focal areas of HER2 overexpression, and common basolateral or lateral membrane staining when evaluated by IHC [4, 12, 14]. Therefore, in the current study, HER2 protein expression levels were assessed according to scoring criteria specific for gastric cancer, which take into account the unique histological features of gastric tumors [4, 12]. The IHC scoring criteria for gastric tumors include not only complete membranous reactivity but also basolateral or lateral membranous reactivity. In addition, the scoring criteria also take into account the variability of staining in this tumor type by not specifying any cutoff for membranous reactivity in biopsy samples; staining in tumor cell clusters of five or more cells is considered positive [14].

In our approach to exploring the impact of variability in staining intensity on overall survival outcomes, we defined variability as $\leq 30 \%$ positively stained cells [14]. Variability was common, and in the overall analyses it did not adversely impact the overall survival benefit gained from the addition of trastuzumab to chemotherapy (although the benefit was numerically lower for patients with variably stained tumors).

Patients with IHC $2+$ and $3+$ scores seemed to derive a benefit from trastuzumab, regardless of staining variability. It should be noted that the patient numbers in the analyzed subgroups were small; therefore, the results should be interpreted with caution. In the IHC 0 and $1+$ groups, analyses were not possible as the subgroups with $>30 \%$ cells stained were too small to assess ( $n=0$ and 10 , respectively). 
Our report of approximately $50 \%$ variability seems to conflict with previously reported rates of up to one-third [20]; however, in this report we assessed variability in HER2 staining intensity/pattern across all IHC scores in all randomized and treated patients, whereas previous studies assessed variability at the highest staining intensity only. Variability in the IHC $3+$ category of ToGA was consistent with the $30 \%$ rate.

Given the frequent occurrence of HER2 staining variability and the slight difference in HER2-positivity rates between biopsy and surgical specimens, it is strongly recommended that a viable number of representative biopsies (ideally 6-8) be collected for HER2 testing, as one or two samples may not give an accurate HER2 result. German guidelines recommend a minimum of eight samples for this reason [21]. Another potential technique for minimizing observer error caused by the variability in staining of gastric tumor material is the use of bright-field HER2 testing methodologies. Bright-field techniques allow for assessment of tumor morphology alongside HER2 evaluation, which permits easier screening of the entire tumor sample and facilitates the identification of focal areas of HER2-positivity [14].

Bang and Van Cutsem et al. showed that the greatest treatment effect was derived in the groups of patients with IHC 2+/FISH-positive and IHC 3+ tumors, while little effect was seen in the IHC 0 and $1+$ groups [12]. We further explored cutoffs for HER2 gene copy number in the IHC 0 and $1+$ categories to potentially identify a patient population with a specific HER2 copy number that may derive a greater benefit from a trastuzumab-based regimen. However, no impact on overall survival was seen when a cutoff of $>6$ HER 2 gene copies was applied, supporting the initial findings that protein expression shows the strongest association with efficacy and should be the initial testing modality to guide treatment decisions (followed by ISH for equivocal IHC $2+$ cases). As sample sizes were low in our assessed sub-subgroup analyses, such findings should be interpreted with caution.

Although HER2 gene amplification was only assessed using FISH in this study, it is anticipated that bright-field ISH techniques may become the preferred assay, although this will vary regionally depending upon the availability of HER2 testing methodologies. A cohort study including gastric tumor samples from the ToGA trial showed that concordance between silver in situ hybridization (SISH) and FISH was $94.5 \%$, further suggesting that alternative ISH methods may be a valid testing option for this tumor type [22]. Similarly, concordance studies in breast cancer, for which CISH or SISH are commonly used in place of FISH, have shown concordance rates of $91 \%$ to $100 \%$ between FISH and CISH and $96 \%$ between FISH and SISH [23-26].
Ring studies, where samples are assessed and compared by several laboratories in a sequential manner, can provide valuable information on interlaboratory IHC and ISH consensus and on factors that may lead to discordant results; in breast cancer, ring studies have been proven to be a successful tool for standardizing HER2 testing and scoring, and a similar approach may be effective in gastric cancer [27].

The addition of trastuzumab to chemotherapy significantly prolongs survival compared with chemotherapy alone in patients with advanced gastric or gastroesophageal junction tumors that show HER2 overexpression or gene amplification (median overall survival: 13.8 months vs. 11.1 months, respectively; HR 0.74; $95 \%$ CI, 0.60-0.91) [12]. An exploratory post hoc analysis of efficacy in two distinct patient subgroups, patients with lower levels of HER2 expression (IHC 0/FISH-positive or IHC 1+/FISHpositive) and patients with higher levels of HER2 expression (IHC $3+$ or IHC 2+/FISH-positive), showed a significant interaction with trastuzumab treatment $(P=0.036)$. A median overall survival of 16.0 months was achieved with trastuzumab plus chemotherapy in patients with higher HER2 expression levels [12], which compares favorably with previous studies of chemotherapy-only regimens in advanced gastric cancer [28, 29]. The current analysis of the ToGA study also showed that all other secondary efficacy endpoints were improved in response to trastuzumab in the higher HER2 expression subgroup.

In conclusion, these results form the largest set of HER2 testing data from patients with gastric or gastroesophageal junction cancer to date and show that HER2 status varies with tumor location and type. The results also serve to validate HER2 as a predictive biomarker in this disease. In 2010, both the EMA and FDA approved trastuzumab treatment based on the overall survival benefit for patients with metastatic gastric or gastroesophageal junction cancer whose tumors have HER2 overexpression, as determined by an accurate and validated assay. Based on the observed survival benefit, trastuzumab, in combination with a platinum salt and fluoropyrimidine-based chemotherapy, is now considered a treatment option in gastric cancer, and it is recommended that all patients with advanced gastric or gastroesophageal junction cancer should be tested for HER2 expression. Approval and registrations have been obtained in other regions worldwide, and patient eligibility for trastuzumab treatment may vary based on local guidelines. Based on the ToGA findings that the subgroup of patients with higher HER2 protein expression gained the greatest survival benefit [12], it is recommended that all patients with gastric or gastroesophageal junction cancer be tested for HER2 to inform treatment decision-making, preferably using IHC as the initial testing modality. As 
gastric cancer is a rapidly progressing disease, a multidisciplinary approach to clinical decision-making is required, involving surgeons, oncologists and pathologists. Access to the HER 2 status of patients is now vital to assist treatment decision-making, ensuring that patients receive the best possible treatment.

Acknowledgments Support for third-party writing assistance for this manuscript, furnished by Daniel Clyde, PhD., was provided by F. Hoffmann-La Roche Ltd. The ToGA study (CenterWatch study ID\#: 147440; Clinical trials.gov ID\#: NCT01041404: http://clinicaltrials. gov/ct2/show/NCT01041404) was sponsored by F. Hoffmann-La Roche Ltd. This study was funded by F. Hoffmann-La Roche Ltd. Eric Van Cutsem has received research funding from F. Hoffmann-La Roche, Ltd., paid to his institution. Yung-Jue Bang has received funding for the ToGA study, paid to his institution, travel support to attend ToGA meetings and editorial support for the preparation of ToGA manuscripts from F. Hoffmann-La Roche Ltd. Timothy Price has participated in an advisory board for and has received a travel grant from F. Hoffmann-La Roche Ltd. Vivian $\mathrm{Ng}$ is an employee of F. Hoffmann-La Roche Ltd./Genentech, Inc., and owns shares in F. Hoffmann-La Roche Ltd. Marlene Thomas is an employee of Roche Diagnostics GmbH. Michaela Lehle is an employee of F. HoffmannLa Roche Ltd. Astrid Kiermaier is an employee of Genentech Inc., and owns shares in F. Hoffmann-La Roche Ltd. Feng Feng-yi, Jian M. $\mathrm{Xu}$, Keun-Wook Lee, Shun-Chang Jiao, Jorge León Chong, Roberto I. López-Sanchez, Oleg Gladkov, Julie Hill, Oliver Stoss, and Josef Rüschoff have no funding to disclose.

Open Access This article is distributed under the terms of the Creative Commons Attribution License which permits any use, distribution, and reproduction in any medium, provided the original author(s) and the source are credited.

\section{References}

1. Slamon DJ, Godolphin W, Jones LA, Holt JA, Wong SG, Keith DE, et al. Studies of the HER-2/neu proto-oncogene in human breast and ovarian cancer. Science. 1989;244:707-12.

2. Gravalos C, Jimeno A. HER2 in gastric cancer: a new prognostic factor and a novel therapeutic target. Ann Oncol. 2008;19: 1523-9.

3. Vogel CL, Cobleigh MA, Tripathy D, Gutheil JC, Harris LN, Fehrenbacher L, et al. Efficacy and safety of trastuzumab as a single agent in first-line treatment of HER2-overexpressing metastatic breast cancer. J Clin Oncol. 2002;20:719-26.

4. Hofmann M, Stoss O, Shi D, Büttner R, van de Vijver M, Kim W, et al. Assessment of a HER2 scoring system for gastric cancer: results from a validation study. Histopathology. 2008;52: 797-805.

5. Park DI, Yun JW, Park JH, Oh SJ, Kim HJ, Cho YK, et al. HER$2 /$ neu amplification is an independent prognostic factor in gastric cancer. Dig Dis Sci. 2006;51:1371-9.

6. Tanner M, Hollmén M, Junttila TT, Kapanen AI, Tommola S, Soini Y, et al. Amplification of HER-2 in gastric carcinoma: association with Topoisomerase IIalpha gene amplification, intestinal type, poor prognosis and sensitivity to trastuzumab. Ann Oncol. 2005;16:273-8.

7. Takehana T, Kunimoto K, Kono K, et al. Status of c-erbB-2 in gastric adenocarcinoma: a comparative study of immunohistochemistry, fluorescence in situ hybridization and enzyme-linked immuno-sorbent assay. Int J Cancer. 2002;98:833-7.
8. Aoyagi K, Kohfuji K, Yano S, Murakami N, Miyagi M, Takeda J. Evaluation of the epidermal growth factor receptor (EGFR) and c-erbB-2 in superspreading type and penetrating-type gastric carcinoma. Kurume Med J. 2001;48:197-200.

9. Brien TP, Depowski PL, Sheehan CE, Ross JS, McKenna BJ. Prognostic factors in gastric cancer. Mod Pathol. 1998;11:870-7.

10. Begnami MD, Fukuda E, Fregnani JH, Nonogaki S, Montagnini AL, Da Costa WL Jr, et al. Prognostic implications of altered human epidermal growth factor receptors (HERs) in gastric carcinomas: HER2 and HER3 are predictors of poor outcome. J Clin Oncol. 2011;29:3030-6.

11. Yoon HH, Shi Q, Sukov WR, Wiktor AE, Khan M, Sattler CA, et al. Association of HER2/ErbB2 expression and gene amplification with pathologic features and prognosis in esophageal adenocarcinomas. Clin Cancer Res. 2012;18:546-54.

12. Bang YJ, Van Cutsem E, Feyereislova A, Chung HC, Shen L, Sawaki A, et al. Trastuzumab in combination with chemotherapy versus chemotherapy alone for treatment of HER2-positive advanced gastric or gastro-oesophageal junction cancer (ToGA): a phase 3, open-label, randomised controlled trial. Lancet. 2010;376:687-97.

13. Lauren $P$. The two histological main types of gastric carcinoma: diffuse and so-called intestinal-type carcinoma. An attempt at a histo-clinical classification. Acta Pathol Microbiol Scand. 1965;64:31-49.

14. Rüschoff J, Hanna W, Bilous M, Hofmann M, Osamura RY, Penault-Llorca F, et al. HER2 testing in gastric cancer: a practical approach. Mod Pathol. 2012;25:637-50.

15. Catalano V, Labianca R, Beretta GD, Gatta G, de Braud F, Van Cutsem E. Gastric cancer. Crit Rev Oncol Hematol. 2000;71:127-64.

16. Waddell T, Verheij M, Allum W, et al. Gastric cancer: ESMOESSO-ESTRO clinical practice guidelines for diagnosis, treatment and follow-up. Ann Oncol. 2013;24 Suppl 6:vi57-63.

17. Kang Y, Bang Y, Lordick F, Park SR, Sawaki A, Chung HC, et al. Incidence of gastric and gastro-esophageal cancer in the ToGA trial: correlation with HER2 positivity. 2008; American Society of Clinical Oncology Gastrointestinal Cancers Symposium (Orlando, FL, USA, 25-27 January): Abstract 11.

18. Shah M, Khanin R, Tang L, Janjigian YY, Klimstra DS, Gerdes $\mathrm{H}$, et al. Molecular classification of gastric cancer: a new paradigm. Clin Cancer Res. 2011;17:2693-701.

19. Rüschoff J. Adenocarcinoma of the GEJ: gastric or oesophageal cancer? Recent Results Cancer Res. 2012;196:107-13.

20. Rüschoff J, Nagelmeier I, Baretton G, Dietel M, Höfler H, Schildhaus HU, et al [Her2 testing in gastric cancer. What is different in comparison to breast cancer?]. Pathologe. 2010;31:208-17.

21. Möhler M, Al-Batran SE, Andus T, Anthuber M, Arends J, Arnold D, et al [German S3-guideline "Diagnosis and treatment of esophagogastric cancer"]. Z Gastroenterol. 2011;49: 461-531.

22. Powell WC, Zielinski D, Ranger-Moore J, Nagelmeier I, Stoss O, Ruschoff J, et al. Determining the HER2 status in gastric cancer: A method comparison study of two patient cohorts. 2010; American Society of Clinical Oncology Gastrointestinal Cancers Symposium (Orlando, FL, USA, 22-24 January): Abstract 17.

23. van de Vijver M, Bilous M, Hanna W, Hofmann M, Kristel P, Penault-Llorca F, et al. Chromogenic in situ hybridisation for the assessment of HER2 status in breast cancer: an international validation ring study. Breast Cancer Res. 2007;9:R68.

24. Hanna WM, Kwok K. Chromogenic in situ hybridization: a viable alternative to fluorescence in situ hybridization in the HER2 testing algorithm. Mod Pathol. 2006;19:481-7.

25. Zhao J, Wu R, Au A, Marquez A, Yu Y, Shi Z. Determination of HER2 gene amplification by chromogenic in situ hybridization 
(CISH) in archival breast carcinoma. Mod Pathol. 2002;15:657-65.

26. Dietel M, Ellis IO, Höfler H, et al. Comparison of automated silver enhanced in situ hybridisation (SISH) and fluorescence ISH (FISH) for the validation of HER2 gene status in breast carcinoma according to the guidelines of the American Society of Clinical Oncology and the College of American Pathologists. Virchows Arch. 2007;451:19-25.

27. Rüschoff J, Dietel M, Baretton G, et al. HER2 diagnostics in gastric cancer-guideline validation and development of standardized immunohistochemical testing. Virchows Arch. 2010;457:299-307.

28. Kang YK, Kang WK, Shin DB, et al. Capecitabine/cisplatin versus 5-fluorouracil/cisplatin as first-line therapy in patients with advanced gastric cancer: a randomised phase III noninferiority trial. Ann Oncol. 2009;20:666-73.

29. Cunningham D, Starling N, Rao S, et al. Capecitabine and oxaliplatin for advanced esophagogastric cancer. N Engl J Med. 2008;358:36-46. 